\title{
Use of a SPAD-502 meter to measure leaf chlorophyll concentration in Arabidopsis thaliana
}

Qihua Ling, Weihua Huang ${ }^{1}$, and Paul Jarvis*

Department of Biology, University of Leicester, University Road, Leicester LE1 7RH, United Kingdom.

${ }^{1}$ Present address: Shanghai Institute of Plant Physiology and Ecology, Shanghai Institutes for Biological Sciences, Chinese Academy of Sciences, 300 Fenglin Road, Shanghai 200032, China.

* To whom correspondence should be addressed

e-mail: $\quad$ rpj3@le.ac.uk

tel.: $\quad+441162231296$

fax: $\quad+441162523330$ 


\begin{abstract}
The SPAD-502 meter is a hand-held device that is widely used for the rapid, accurate and non-destructive measurement of leaf chlorophyll concentrations. It has been employed extensively in both research and agricultural applications, with a range of different plant species. However, its utility has not been fully exploited in relation to the most intensively studied model organism for plant science research, Arabidopsis thaliana. Measurements with the SPAD-502 meter produce relative SPAD meter values that are proportional to the amount of chlorophyll present in the leaf. In order to convert these values into absolute units of chlorophyll concentration, calibration curves must be derived and utilized. Here, we present calibration equations for Arabidopsis that can be used to convert SPAD values into total chlorophyll per unit leaf area $\left(\mathrm{nmol} / \mathrm{cm}^{2} ; \mathrm{R}^{2}=0.9960\right)$ or per unit fresh weight of leaf tissue ( $\mathrm{nmol} / \mathrm{mg} ; \mathrm{R}^{2}=0.9809$ ). These relationships were derived using a series of Arabidopsis chloroplast biogenesis mutants that exhibit chlorophyll deficiencies of varying severity, and were verified by the subsequent analysis of senescent or light-stressed leaves. Our results revealed that the converted SPAD values differ from photometric measurements of solventextracted chlorophyll by just $\sim 6 \%$ on average.
\end{abstract}

\title{
Keywords
}

Arabidopsis; Calibration curve; Chlorophyll content; Chloroplast development; SPAD meter 


\section{Introduction}

Leaf chlorophyll concentration is an important parameter that is frequently measured as an indicator of chloroplast development, photosynthetic capacity, leaf nitrogen content, or general plant health. In the laboratory, it is commonly determined photometrically following extraction of the pigments using an organic solvent, such as acetone or dimethyl formamide (Arnon 1949; Porra et al. 1989). While this method is well-established and accurate, it is timeconsuming, destructive (the leaf material must be excised from the plant, and is lost), and necessitates the use of toxic or flammable chemicals. The SPAD-502 meter (Konica-Minolta, Japan) provides an alternative method for the measurement of relative leaf chlorophyll levels that overcomes these disadvantages. It is an inexpensive, hand-held device based on two light-emitting diodes and a silicon photodiode receptor, that measures leaf transmittance in the red $(650 \mathrm{~nm}$; the measuring wavelength) and infrared $(940 \mathrm{~nm}$; a reference wavelength used to adjust for non-specific differences between samples) regions of the electromagnetic spectrum. These transmittance values are used by the device to derive a relative SPAD meter value (typically between 0.0 and 50.0) that is proportional to amount of chlorophyll in the sample (Uddling et al. 2007). The meter has been used extensively in both research and agricultural settings, and there are many publications in the scientific literature that describe its use.

In order to convert relative SPAD meter values into units of absolute chlorophyll concentration, it is necessary to employ a calibration curve (Markwell et al. 1995). The relationship between SPAD values and chlorophyll concentration has been investigated in a variety of different species, and has been found to display considerable interspecific variation (Castelli et al. 1996; Uddling et al. 2007). This variability is presumed to be due to structural differences between the leaves of different species, causing different light reflection or scattering effects. Thus, in order for the SPAD-502 meter to be used to record real chlorophyll concentration values with reasonable accuracy, a calibration equation must be derived specifically for the particular species of interest (Richardson et al. 2002). Arabidopsis thaliana has been widely adopted across the globe as a model organism for studies on plant development, cellular and molecular biology, and biochemistry. The particular advantages of this species are well documented, and include its completely-sequenced genome, diminutive stature, and rapid generation time, as well as the availability of extensive collections of insertional mutants such that it is possible to quickly identify a knockout for almost any gene 
of interest. As a result, a considerable proportion of the chloroplast and plastid research that is undertaken today is conducted using the Arabidopsis model system (Leister 2003; López-Juez 2007; Sakamoto et al. 2008). Nonetheless, only a very small number of Arabidopsis studies have employed the SPAD-502 meter, and these have either presented the data in unconverted arbitrary units (Stettler et al. 2009) or utilized a crude scaling factor to estimate actual chlorophyll content (Takami et al. 2010). To enable the advantages of the SPAD meter to be exploited more fully in the field of Arabidopsis research, we have derived specific calibration relationships that can be used to convert SPAD values accurately into absolute chlorophyll concentration values, on either a per-leaf-area or a per-fresh-weight basis.

\section{Materials and methods}

Plant material and growth conditions

All plants used in this study were Arabidopsis thaliana of the Columbia-0 ecotype. The +/tic110-1, hsp93-V-1, tic40-4, ppil-1 and hsp93-V-1 hsp93-III-1 (hsp93-V/III) mutants employed to derive the calibration relationships have all been described previously (Jarvis et al. 1998; Kovacheva et al. 2005; Kovacheva et al. 2007). Similarly, the pph-1 and paol mutants have been presented in earlier reports (Pruzinska et al. 2005; Schelbert et al. 2009).

Plants were germinated on MS medium and allowed to grow for 10 days for prior to transferral to soil, or sown on soil directly. They were grown in $\sim 100 \mu \mathrm{mol} / \mathrm{m}^{2} / \mathrm{s}$ white light under a long-day cycle (16-hours-light/8-hours-dark), as described previously (Aronsson and Jarvis 2002).

Dark and light-stress treatments

Dark treatments for the induction of senescence were conducted using two different methods, as described previously (Pruzinska et al. 2005; Schelbert et al. 2009). In the first method (Fig. 2), developmentally-equivalent leaves were detached from a plant and placed on wet filter paper in sealed Petri dishes. The dishes were then placed in a dark container within a growth cabinet for six days. In the second method (Fig. 3), the leaves were carefully wrapped in 
aluminium foil whilst still attached to the plant, and then left under standard growth conditions for 4-6 days.

Light stress treatments involved exposure of 21-day-old plants grown under standard conditions to high-intensity white light $\left(\sim 2000 \mu \mathrm{mol} / \mathrm{m}^{2} / \mathrm{s}\right)$, delivered through a chilled-water heat sink, for three hours per day for seven days. After the final period of high-light treatment, the plants were returned to standard growth conditions for 24 hours prior to conducting the measurements.

Chlorophyll measurements and data analysis

Absolute chlorophyll concentration measurements were conducted using a spectrophotometer and dimethyl formamide extracts of leaf tissue, as described previously (Constan et al. 2004; Porra et al. 1989). Leaf tissue for these measurements was harvested using a circular punch (cork borer) that yields $0.5 \mathrm{~cm}$ diameter leaf discs that are $0.19635 \mathrm{~cm}^{2}$ in area; excised discs were also weighed, enabling the chlorophyll data to be expressed in relation to fresh weight as well as leaf area. Each solvent extract contained several leaf discs from multiple different plants per genotype or treatment. SPAD values were recorded using exactly the same leaves from the same plants, prior to sampling, using a SPAD-502 meter (Konica-Minolta, Japan). Twelve independent SPAD measurements were made per genotype or treatment, using several different plants.

Relationships between the two datasets were analysed using both SigmaPlot (SPSS Science Inc.) and Excel (Microsoft) software. Several models were tested and the two packages gave identical results, indicating that second-order polynomial functions provide the best fit.

\section{Results and discussion}

In order to derive useful relationships between SPAD meter readings and chlorophyll content, it is necessary to study plants that have a variety of different pigmentation levels. To this end, we exploited a range of well-characterized chloroplast biogenesis mutants that are defective in the import of proteins into plastids (Jarvis 2008). The selected mutant genotypes (+/tic110, 
hsp93-V, tic40, ppil and hsp93-V/III) exhibit varying degrees of chlorosis due to variations in the importance of the affected genes (Fig. 1a), and were analysed alongside wild-type plants of two different ages. All of the plants were used contemporaneously to make both SPAD meter readings and photometric measurements using pigment extracts. By plotting these two datasets against each other, we assessed possible relationships between them. While linear and exponential relationships between SPAD values and chlorophyll concentrations have previously been proposed (Uddling et al. 2007), we observed a much stronger fit using second-order polynomial functions (Fig. 1, b and c); $\mathrm{R}^{2}$ values for linear and exponential relationships were, respectively, 0.979 and 0.973 for the data in $b$, and 0.959 and 0.972 for the data in c, and thus significantly lower than those shown in Fig. 1 for the selected polynomial functions. Others have similarly reported that such polynomial functions best describe the relationship between SPAD values and chlorophyll concentration (Hawkins et al. 2009; Markwell et al. 1995).

To test the utility of the calibration equations presented in Fig. 1, we studied plants exhibiting dark-induced senescence. Studying such environmentally induced chlorosis enabled us to assess whether the aforementioned relationships (derived based on an analysis of genetically induced chlorosis) would remain valid under different conditions. Two different methods of dark treatment for the induction of senescence have been described in the literature: the incubation of detached leaves in a dark container, and the covering of attached leaves with aluminium foil (Pruzinska et al. 2005; Schelbert et al. 2009). We elected to employ both of these methods, starting with the former. In addition to wild type, we incorporated two well-characterized, senescence-defective mutants in our analysis, as additional controls. These were the pheophytinase (PPH) mutant, $p p h$, and the pheide $a$ oxygenase (PAO) mutant, paol, both of which are deficient in an important step of chlorophyll breakdown and, therefore, express a "stay-green" phenotype under conditions that normally induce senescence (Pruzinska et al. 2005; Schelbert et al. 2009).

After six days in the dark, detached wild-type leaves were pale-yellow in appearance, whereas pph and paol mutant leaves retained considerable amounts of green pigmentation (Fig. 2a). While the pph leaves were uniformly green, paol leaves had a patchy appearance with both green and yellow sectors. This phenotypic difference, although not specifically reported previously, presumably reflects the fact that pheide $a$ and red chlorophyll catabolites accumulate in paol, but not in $p p h$, which can cause a lesion-mimic or cell death phenotype in the former mutant in the light (Schelbert et al. 2009). As a result of its patchy appearance, paol was deemed unsuitable for the purposes of our experiment (as it would have been 
difficult to obtain consistent measurements), and so we focused only on the wild type and $p p h$. Dark-treated and control (untreated) leaves were used to make both SPAD meter readings and photometric measurements using pigment extracts. The SPAD data were converted to chlorophyll values employing the calibration equations displayed in Fig. 1, and then compared with photometrically determined data. As shown in Fig. 2 (b and c), the two datasets were very similar, indicating that the derived conversion formulae are suitable for the measurement of chlorophyll under different conditions.

In relation to chlorophyll $a / b$ ratios, it should be noted that the meter's measuring light has a peak wavelength of $650 \mathrm{~nm}$, which lies approximately midway between the absorption maxima of chlorophylls $a$ and $b$, and in fact is the point of intersection of the absorption spectra of the two pigments (Arnon 1949; Porra et al. 1989). Thus, while caution is advised, measurements with the SPAD meter are unlikely to be strongly distorted by $a / b$ ratio changes. Indeed, the effect of the $p p h$ mutation is such that, under dark-treatment conditions, the chlorophyll $a / b$ ratio is dramatically reduced in the mutant relative to wild type (data not shown; Schelbert et al. 2009). The fact that SPAD readings taken using dark-treated $p p h$ plants were similar to corresponding photometric measurements (Fig. 2, b and c) supports the notion that the meter is not adversely influenced by deviations in the chlorophyll $a / b$ ratio.

Using the second method for the induction of senescence, we observed that most leaves covered for six days would dry out and die. The covering period was therefore reduced to four days, and under these conditions we observed the expected chlorosis in wild type, and a "stay-green" phenotype in pph (Fig. 3a). However, paol leaves were not viable under these conditions either, and, as with the detached leaves (Fig. 2), this difference from $p p h$ may be due the accumulation of harmful chlorophyll catabolites in the paol mutant (Pruzinska et al. 2005; Schelbert et al. 2009). Importantly, analysis of chlorophyll levels in these leaves using the two different methods again revealed closely correlated datasets (Fig. 3b), supporting the utility of the SPAD conversion formulae.

In a final experiment to corroborate the usefulness of the calibration equations, we analysed light-stressed plants simultaneously using both chlorophyll quantification methods. Prolonged exposure to high light intensities of a plant not acclimated to such conditions leads to significant damage of the photosynthetic apparatus and a concomitant depletion of photosynthetic pigments (Aronsson et al. 2006; Powles 1984). As expected, the light-stressed plants appeared paler than control plants, and somewhat stunted (Fig. 4a). Comparison of the converted SPAD data with those derived photometrically from extracted pigment confirmed the suitability of the formulae for assaying chlorophyll content differences induced by another, 
different set of conditions (Fig. 4b). Considering all of the data shown in Figs. 2, 3 and 4, it is apparent that the values determined using the SPAD meter differ from the conventionally acquired data by $\sim 6 \%$, on average, which for most purposes should be an acceptable margin of error. It is noteworthy that there is a strong linear relationship between readings of the SPAD-502 meter, employed here, and another popular chlorophyll meter, the N-Tester (Yara, Norway; formerly Hydro) (Levey and Wingler 2005; Uddling et al. 2007). Thus, the conversion formulae derived here may also be useful in conjunction with data from that device.

It should be noted that SPAD meter readings can be influenced by changing growth conditions that, for example, may lead to a redistribution of chloroplasts within mesophyll cells (Naus et al. 2010). For this reason, it is advisable to always include an internal control in each experiment, as a point of reference, and to be cautious about making comparisons between experiments conducted on different occasions. Nonetheless, provided that the plants to be measured are grown under the commonly-used and very standard, controlled conditions employed here, the results should be reproducible. Another consideration that should be borne in mind is the size of the meter's measuring area, which is $2 \mathrm{~mm} \times 3 \mathrm{~mm}$. While this compactness is an advantage in that it may accommodate all but the smallest leaves of rosettestage plants, it may be disadvantageous in relation to leaves that have an excessively patchy or reticulate appearance (e.g., paol; Fig. 2a). However, this problem may be at least partially overcome by taking multiple measurements using different areas of the same leaf.

\section{Conclusion}

The formulae presented here are suitable for converting SPAD readings into values of chlorophyll concentration in Arabidopsis leaves, and the converted values correlate closely with direct photometric measurements of extracted chlorophyll. Use of the SPAD-502 meter is a very quick and simple procedure, requiring no toxic or flammable solvents. Moreover, as the procedure is non-destructive, this approach has significant advantages in relation to experiments that require the recovery of biological material from the leaves (e.g., nucleic acids or protein), the analysis of particularly precious plants that can neither be sacrificed nor damaged, and studies where it is desirable to monitor dynamic changes of chlorophyll content in the same individual throughout development or in responses to changing conditions. 


\section{Acknowledgements}

This work was supported by a Royal Society International Incoming Fellowship (to WH), the Royal Society Rosenheim Research Fellowship (to PJ), and by Biotechnology and Biological Sciences Research Council (BBSRC) grants BB/D016541/1, BB/F020325/1 and BB/H008039/1 (to PJ). 


\section{Figure legends}

Fig. 1. Use of chloroplast biogenesis mutants to derive calibration relationships between SPAD meter readings and photometric chlorophyll measurements.

(a) Arabidopsis plants of the indicated genotypes were grown side-by-side under identical conditions for either 35 days (wild type and all mutants) or 45 days (wild type only), and then typical individuals were selected for photography.

(b, c) Plants similar to those shown in (a) were used to make SPAD meter readings, and to make direct measurements of absolute chlorophyll concentration by the photometric analysis of solvent extracts. The SPAD values are plotted along the x-axis, and the corresponding chlorophyll concentration values are plotted along the y-axis (nmol chlorophyll $a+b$ per $\mathrm{cm}^{2}$ leaf area in [b]; nmol chlorophyll $a+b$ per mg leaf fresh weight in [c]). The presented data are means $( \pm \mathrm{SD})$ derived from 12 independent SPAD meter readings done on up to 12 different plants, or from three independent solvent extracts (one less for tic40), each one containing four leaf discs from up to four different plants. Second order polynomial functions were found to fit the data most closely; the relevant curves and equations, and corresponding $\mathrm{R}^{2}$ values, are shown in each panel.

Fig. 2. Analysis of dark-induced senescence in detached leaves for the validation of the calibration equations.

(a) Developmentally equivalent leaves of 35-day-old wild-type and "stay-green" mutant (pph and paol) plants were detached, incubated on wet filter paper in a dark container for six days, and then photographed.

$(b, c)$ Leaves similar to those shown in (a) were used to make both SPAD meter readings and photometric measurements of chlorophyll concentration using extracted pigments. The SPAD readings were converted to chlorophyll concentration values using the relationships presented in Fig. 1, and then the two datasets were plotted side-by-side for comparison purposes. Values shown are means $( \pm \mathrm{SD})$ derived from 12 independent SPAD meter readings done on six different plants, or from three independent solvent extracts, each one containing four leaf discs from two different plants. 
Fig. 3. Analysis of dark-induced senescence in attached leaves for the validation of the calibration equations.

(a) Developmentally equivalent leaves of 35-day-old wild-type and "stay-green" mutant (pph and paol) plants were covered with aluminium foil (whilst still attached to the plant), and then left for four days under standard growth conditions prior to detachment and photography. (b) Leaves similar to those shown in (a) were used to make both SPAD meter readings and photometric measurements of chlorophyll concentration using extracted pigments; the SPAD readings were converted to chlorophyll concentration values using the relationships in Fig. 1 prior to analysis. Values shown are means $( \pm \mathrm{SD})$ derived from 12 independent SPAD meter readings done on six different plants, or from three independent solvent extracts, each one containing four leaf discs from two different plants.

Fig. 4. Analysis of light-stressed plants for the validation of the calibration equations.

(a) Wild-type Arabidopsis plants were grown under standard conditions for 21 days, and then subjected to periods of high-light stress $\left(2000 \mu \mathrm{mol} / \mathrm{m}^{2} / \mathrm{s}\right)$ over a seven day period. Typical stressed and control (untreated) plants are shown.

(b) Developmentally equivalent leaves from plants similar to those shown in (a) were used to make both SPAD meter readings and photometric measurements of chlorophyll concentration using extracted pigments; the SPAD readings were converted to chlorophyll concentration values using the relationships in Fig. 1 prior to analysis. Values shown are means $( \pm \mathrm{SD})$ derived from 12 independent SPAD meter readings done on three different plants, or from three independent solvent extracts, each one containing four leaf discs from a single plant. 


\section{References}

Arnon DI (1949) Copper enzymes in isolated chloroplasts. Polyphenoloxidase in Beta vulgaris. Plant Physiol. 24:1-15

Aronsson H, Jarvis P (2002) A simple method for isolating import-competent Arabidopsis chloroplasts. FEBS Lett. 529:215-220

Aronsson H, Combe J, Patel R, Jarvis P (2006) In vivo assessment of the significance of phosphorylation of the Arabidopsis chloroplast protein import receptor, atToc33. FEBS Lett. 580:649-655

Castelli F, Contillo R, Miiceli F (1996) Non-destructive determination of leaf chlorophyll content in four crop species. J. Agron. Crop Sci. 177:275-283

Constan D, Patel R, Keegstra K, Jarvis P (2004) An outer envelope membrane component of the plastid protein import apparatus plays an essential role in Arabidopsis. Plant J. 38:93-106

Hawkins TS, Gardiner ES, Comer GS (2009) Modeling the relationship between extractable chlorophyll and SPAD-502 readings for endangered plant species research. J. Nat. Conserv. 17:123-127

Jarvis P (2008) Targeting of nucleus-encoded proteins to chloroplasts in plants (Tansley Review). New Phytol. 179:257-285

Jarvis P, Chen LJ, Li H, Peto CA, Fankhauser C, Chory J (1998) An Arabidopsis mutant defective in the plastid general protein import apparatus. Science 282:100-103

Kovacheva S, Bédard J, Wardle A, Patel R, Jarvis P (2007) Further in vivo studies on the role of the molecular chaperone, Hsp93, in plastid protein import. Plant J. 50:364-379

Kovacheva S, Bédard J, Patel R, Dudley P, Twell D, Ríos G, Koncz C, Jarvis P (2005) In vivo studies on the roles of Tic110, Tic40 and Hsp93 during chloroplast protein import. Plant J. 41:412-428

Leister D (2003) Chloroplast research in the genomic age. Trends Genet. 19:47-56.

Levey S, Wingler A (2005) Natural variation in the regulation of leaf senescence and relation to other traits in Arabidopsis. Plant Cell Environ. 28:223-231

López-Juez E (2007) Plastid biogenesis, between light and shadows. J. Exp. Bot. 58:11-26

Markwell J, Ostermann JC, Mitchell JL (1995) Calibration of the Minolta SPAD-502 leaf chlorophyll meter. Photosynth Res. 46:467-471

Naus J, Prokopova J, Rebicek J, Spundova M (2010) SPAD chlorophyll meter reading can be pronouncedly affected by chloroplast movement. Photosynth Res.:DOI 10.1007/s11120-11010-19587-z

Porra RJ, Thompson WA, Kriedemann PE (1989) Determination of accurate extinction coefficients and simultaneous equations for assaying chlorophylls $a$ and $b$ extracted with four different solvents: verification of the concentration of chlorophyll standards by atomic absorption spectroscopy. Biochim. Biophys. Acta 975:384-394

Powles SB (1984) Photoinhibition of photosynthesis induced by visible light. Annu. Rev. Plant Physiol. 35:15-44

Pruzinska A, Tanner G, Aubry S, Anders I, Moser S, Muller T, Ongania KH, Krautler B, Youn JY, Liljegren SJ, Hortensteiner S (2005) Chlorophyll breakdown in senescent Arabidopsis leaves. Characterization of chlorophyll catabolites and of chlorophyll catabolic enzymes involved in the degreening reaction. Plant Physiol. 139:52-63

Richardson AD, Duigan SP, Berlyn GP (2002) An evaluation of noninvasive methods to estimate foliar chlorophyll content. New Phytol. 153:185-194 
Sakamoto W, Miyagishima S, Jarvis P (2008) Chloroplast biogenesis: control of plastid development, protein import, division and inheritance. In The Arabidopsis Book, R. Last, ed (Rockville, MD, USA: American Society of Plant Biologists), pp. 1-30

Schelbert S, Aubry S, Burla B, Agne B, Kessler F, Krupinska K, Hörtensteiner S (2009) Pheophytin pheophorbide hydrolase (pheophytinase) is involved in chlorophyll breakdown during leaf senescence in Arabidopsis. Plant Cell 21:767-785

Stettler M, Eicke S, Mettler T, Messerli G, Hörtensteiner S, Zeeman SC (2009) Blocking the metabolism of starch breakdown products in Arabidopsis leaves triggers chloroplast degradation. Mol. Plant 2:1233-1246

Takami T, Shibata M, Kobayashi Y, Shikanai T (2010) De novo biosynthesis of fatty acids plays critical roles in the response of the photosynthetic machinery to low temperature in Arabidopsis. Plant Cell Physiol. 51:1265-1275

Uddling J, Gelang-Alfredsson J, Piikki K, Pleijel H (2007) Evaluating the relationship between leaf chlorophyll concentration and SPAD-502 chlorophyll meter readings. Photosynth Res. 91:37-46 
Figure 1

Click here to download high resolution image

(a)

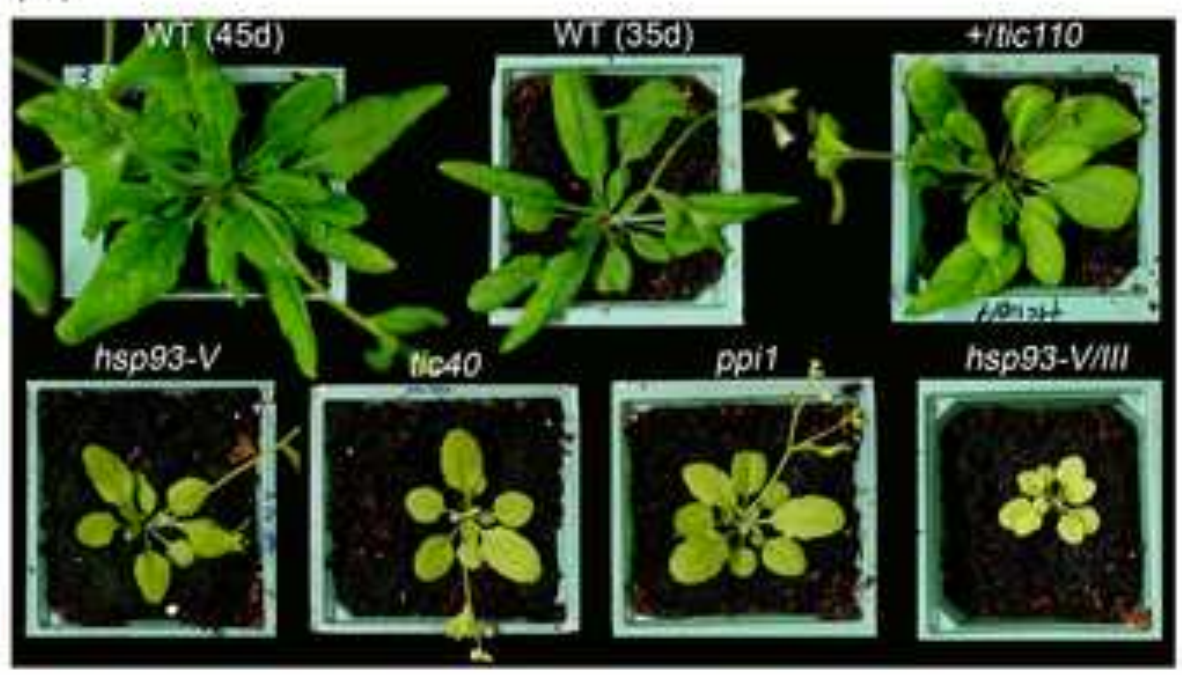

(b)

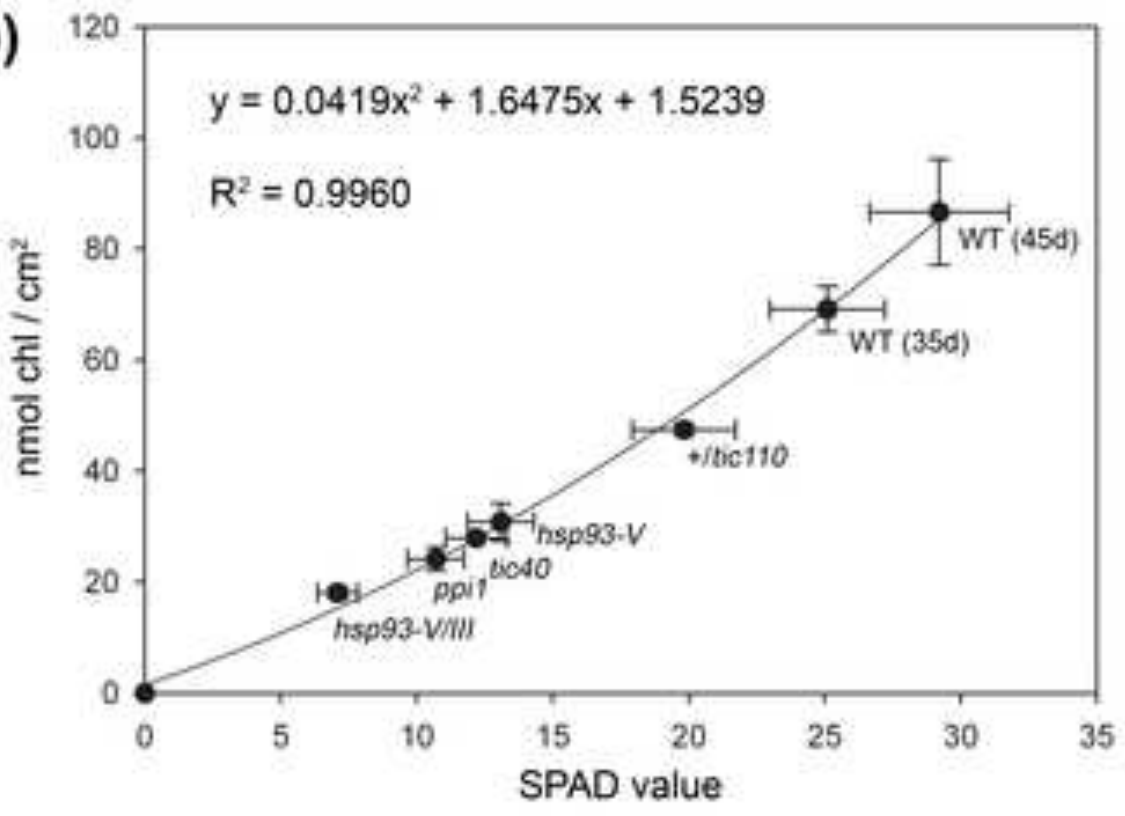

(c)

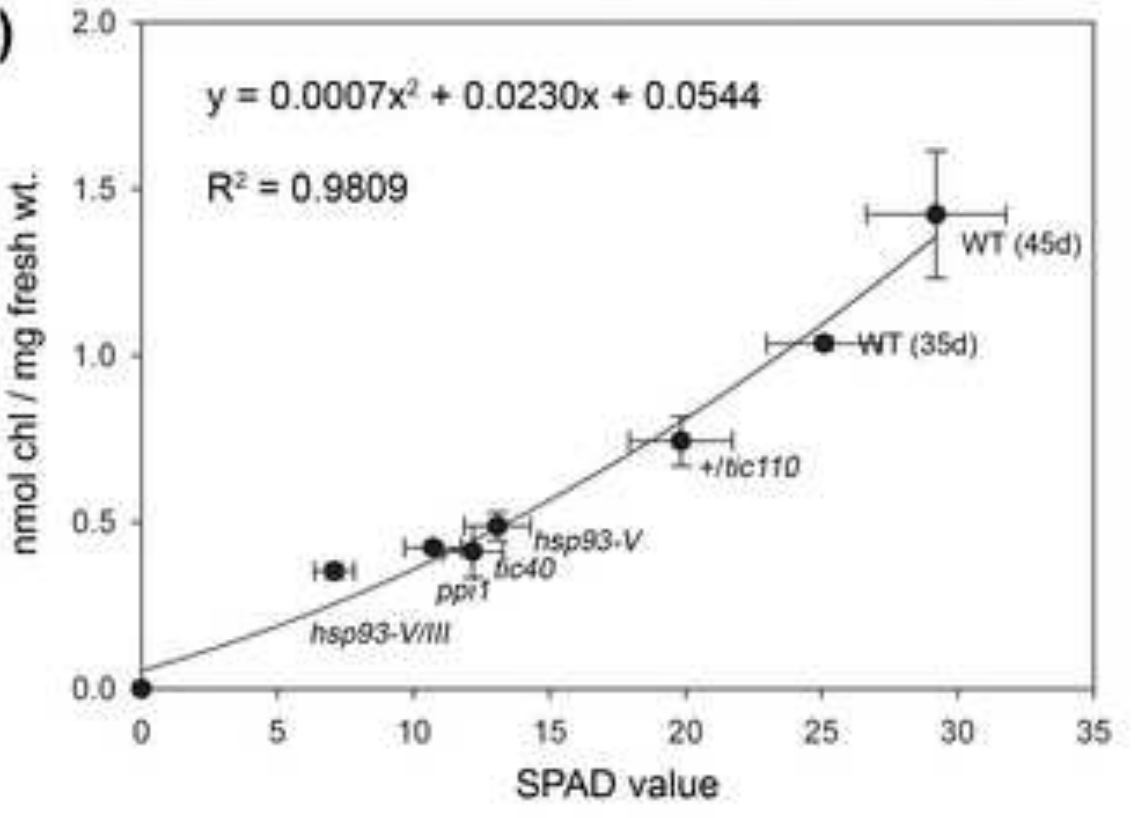


Figure 2

Click here to download high resolution image

(a)

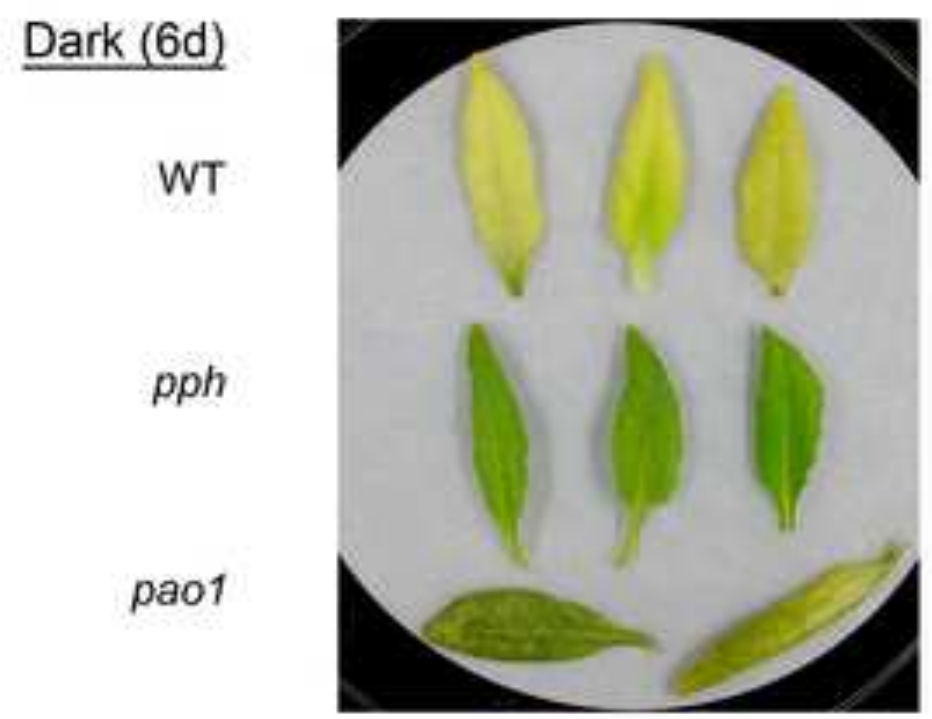

(b)

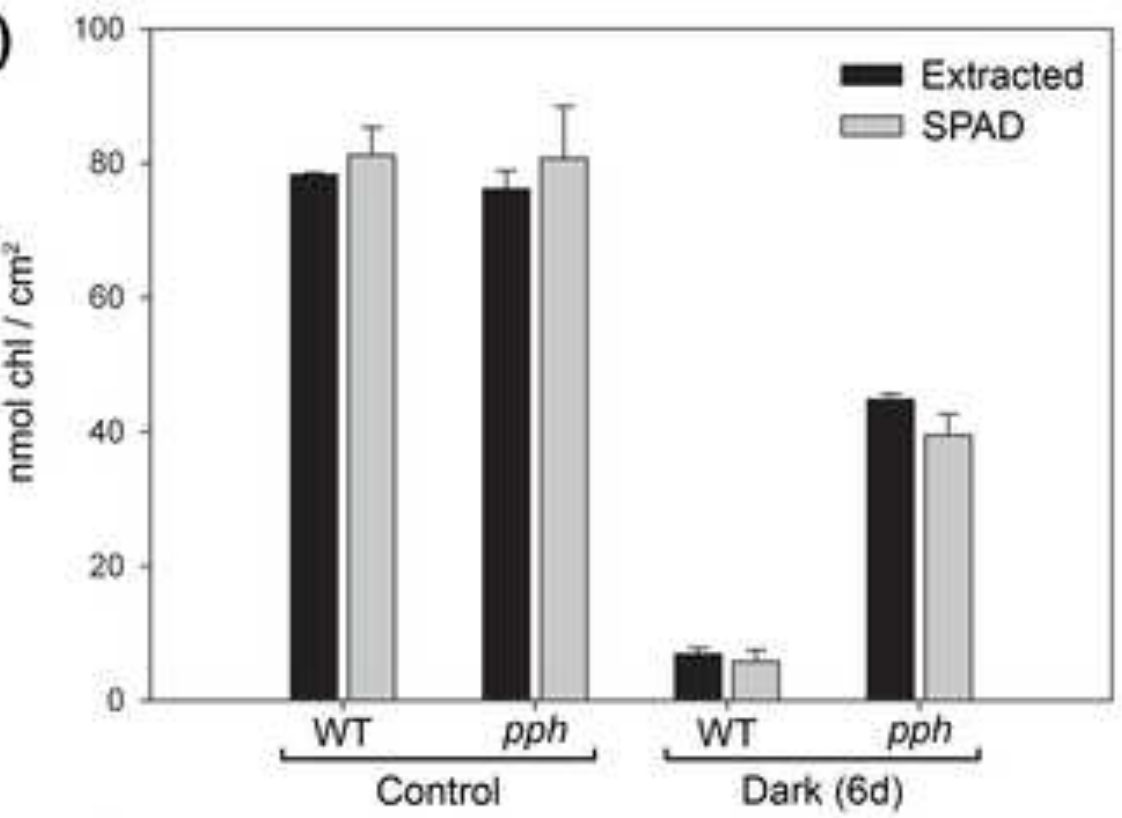

(c)

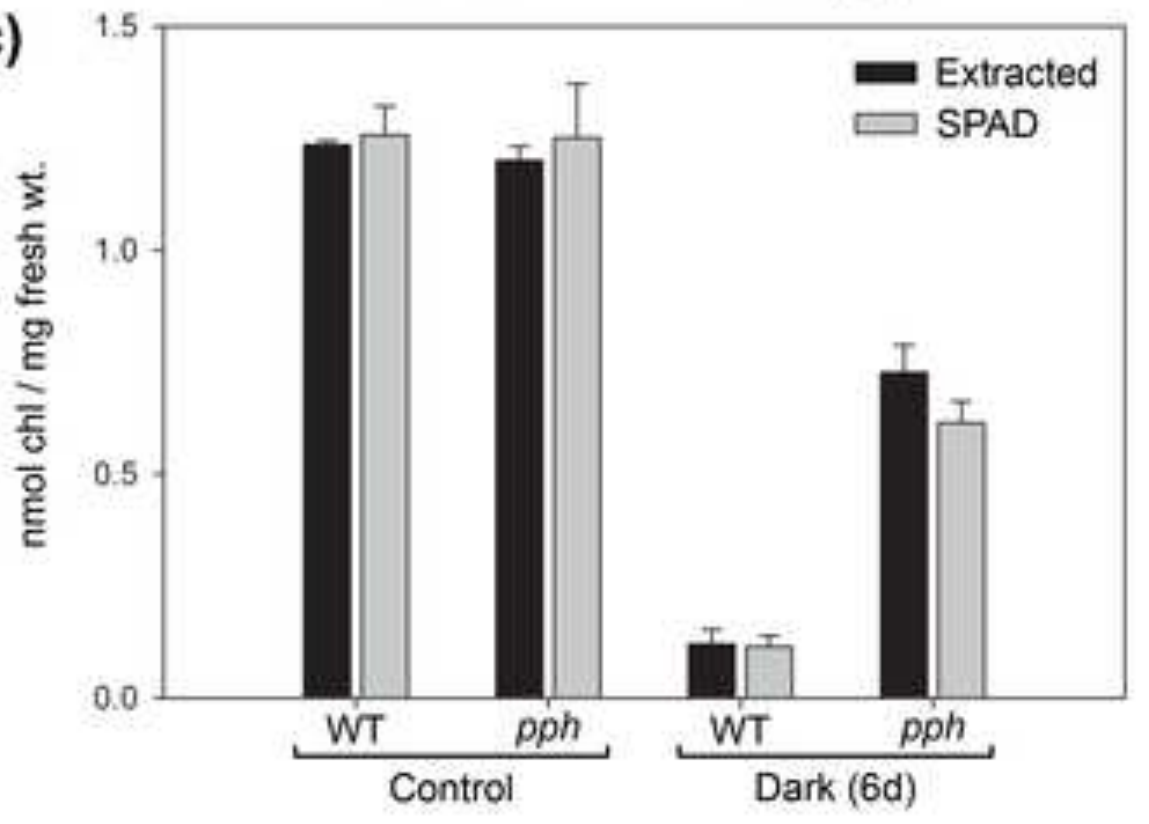


(a) $\underline{\operatorname{Dark}(4 \mathrm{~d})}$

WT

pph

pao1

(b)

(a) Light stress

Control

Stressed

(b)
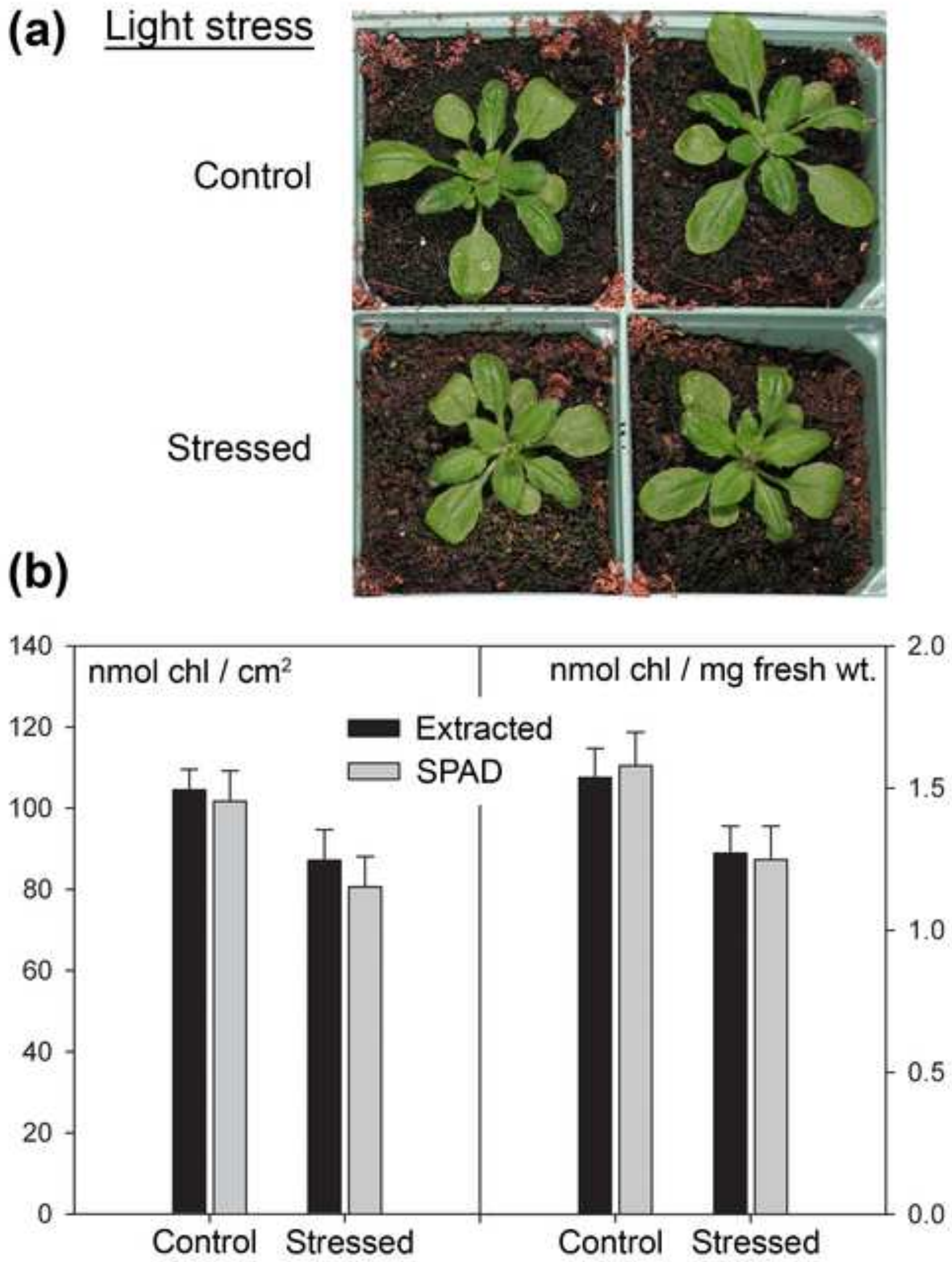


\section{Erratum to: Use of a SPAD-502 meter to measure leaf chlorophyll concentration in Arabidopsis thaliana}

Photosynth. Res. (2011) 107:209-214

DOI 10.1007/s11120-010-9606-0

Qihua Ling, Weihua Huang ${ }^{1}$, and Paul Jarvis*

Department of Biology, University of Leicester, University Road, Leicester LE1 7RH, United Kingdom.

${ }^{1}$ Present address: Institute of Plant Physiology and Ecology, Shanghai Institutes for Biological Sciences, Chinese Academy of Sciences, 300 Fenglin Road, Shanghai 200032, China.

* To whom correspondence should be addressed

\begin{tabular}{|c|c|}
\hline e-mail: & rpj3@le.ac.uk \\
\hline tel.: & +441162231296 \\
\hline $\mathrm{x}$ : & +441162523 \\
\hline
\end{tabular}


Due to the omission of a scaling factor of 4 from the chlorophyll per leaf area calculations, all values with units of $\mathrm{nmol} / \mathrm{cm}^{2}$ were fourfold higher than they should have been. This affected the y-axis values in Figs. 1b, 2b, 3b (left side) and 4b (left side); the maximal y-axis values should be 30, 25, 15 and 35, respectively. Most importantly, the equation in Fig. 1b should be:

$y=0.0105 x^{2}+0.4119 x+0.3810$

None of the chlorophyll per fresh weight data are affected by this erratum, nor is the running text influenced in any way. All $R^{2}$ values are unaffected. 